Case Report

\title{
A Case of Polymyxin b-Immobilized Fiber Column Treatment for Rapidly Progressive Interstitial Pneumonia Associated with Clinically Amyopathic Dermatomyositis
}

\author{
Oh Sasaki, Makoto Dohi, Hiroaki Harada, Mitsuru Imamura, Yumi Tsuchida, \\ Kensuke Yamaguchi, Toshihiko Komai, and Kazuhiko Yamamoto \\ Department of Allergy and Rheumatology, Graduate School of Medicine, University of Tokyo, Hongo 7-3-1, Bunkyo-ku, \\ Tokyo 113-0033, Japan \\ Correspondence should be addressed to Makoto Dohi; mdohi-tky@umin.ac.jp
}

Received 12 April 2013; Revised 23 June 2013; Accepted 8 July 2013

Academic Editor: Raed Dweik

Copyright (C) 2013 Oh Sasaki et al. This is an open access article distributed under the Creative Commons Attribution License, which permits unrestricted use, distribution, and reproduction in any medium, provided the original work is properly cited.

\begin{abstract}
We report a case of rapidly progressive interstitial pneumonia associated with clinically amyopathic dermatomyositis who responded to single course of polymyxin b-immobilized fiber column treatment. Initial treatment with pulsed corticosteroids and cyclophosphamide, intravenous immunoglobulin, and cyclosporine seemed to suppress the activity of interstitial lung disease temporarily, but signs of relapse were detected such as elevation of serum KL-6 level and progressing pulmonary shadows in chest computed tomography scan. After polymyxin b-immobilized fiber column treatment, the areas of pulmonary shadows drastically decreased. Gradually, arterial partial pressure of oxygen/fraction of inspired oxygen $\left(\mathrm{PaO}_{2} / \mathrm{FiO}_{2}\right)$ ratio recovered, and serum ferritin level and KL-6 level decreased. These findings indicate that polymyxin b-immobilized fiber column treatment could be promising in combination with conventional therapy for rapidly progressive interstitial pneumonia associated with clinically amyopathic dermatomyositis, especially at the early phase of relapse.
\end{abstract}

\section{Introduction}

Dermatomyositis (DM) is an idiopathic connective tissue disease which is characterized by inflammation of the muscles and the skin. It is also a systemic disease involving the joints, esophagus, lungs, and heart. Clinically amyopathic dermatomyositis (C-ADM) is characterized by typical cutaneous manifestations and minor involvement of skeletal muscles [1]. It is well known that the interstitial lung disease (ILD) associated with C-ADM often presents with rapid onset and progression [2] and that the type of ILD is resistant to conventional therapy and has a poor prognosis especially in East Asia [3, 4].

It has been reported that direct hemoperfusion with polymyxin b-immobilized fiber column (PMX) effectively removed circulating endotoxin both in vitro and in vivo studies [5]. It was initially developed as a treatment for sepsis, and it has also revealed favorable effects on arterial partial pressure of oxygen/fraction of inspired oxygen $\left(\mathrm{PaO}_{2} / \mathrm{FiO}_{2}\right)$ ratio in acute respiratory failure [6]. Here, we describe a patient of rapidly progressive interstitial pneumonia associated with C-ADM whose relapse after intensive conventional therapy was successfully treated with PMX hemoperfusion.

\section{Case Report}

A 37-year-old man presented with skin rash for the last two months. He also had progressing dyspnea for the last two weeks and visited a hospital to consult with a physician. His oxygen saturation $\left(\mathrm{SpO}_{2}\right)$ was as low as $91 \%$ at room air in resting state, and he was admitted to the hospital. He had skin rashes in the face and on the hands. Chest computed tomography (CT) scan revealed nonsegmental pulmonary infiltrate and grand-glass opacity mainly distributed in the subpleural areas of the lower lobes. In addition, there were some legions of consolidation with air bronchogram in the peripheral lung, which implied traction bronchiectasis. The bronchoalveolar 
lavage fluid (BALF) analysis showed increases in total cells $\left(2.25 \times 10^{5} / \mathrm{mL}\right)$ and lymphocyte fraction $(64.5 \%)$. Culture of BALF was negative. In transbronchial lung biopsy samples, lymphocytes and macrophages was infiltrated, and masson body-like fibrotic foci was formed.

He was suspected of rapidly progressing interstitial lung disease with dermatomyositis and transferred to our hospital. He had Gottron's papules, mechanic's hands, and spotted rashes between eyebrows, but he did not have heliotrope rash or other rashes on joints or body trunk. Manual muscle testing revealed no muscle weakness both in proximal and distal muscles. Fine crackles were audible in the bilateral lower lung fields. Laboratory findings on admission revealed a white blood cell count of $4,200 / \mathrm{mm}^{3}$ with $76.1 \%$ neutrophils C-reactive protein was slightly elevated $(3.21 \mathrm{mg} / \mathrm{dL}$ [standard value; $0-0.4 \mathrm{mg} / \mathrm{dL}$ ]). His serum lactate dehydrogenase (LDH), KL-6, ferritin, aldolase, and D-Dimer levels were elevated (586 IU/L [119-229 IU/L]), $1835 \mathrm{U} / \mathrm{mL}$ [<500 U/mL], $842 \mathrm{ng} / \mathrm{mL} \quad[20-280 \mathrm{ng} / \mathrm{mL}], 14.1 \mathrm{U} / \mathrm{L}$ [2.7$7.5 \mathrm{U} / \mathrm{L}]$, and $12.4 \mu \mathrm{g} / \mathrm{mL}[<1.0 \mu \mathrm{g} / \mathrm{mL}]$, resp.), but creatine kinase (CK) was not elevated (201 U/L [55-210 U/L]). He did not have antinuclear antibody, anti-Jo-1 antibody, or other anti-aminoacyl-tRNA synthetase antibodies. Immunoprecipitation with the patient's serum revealed that he had a $140 \mathrm{kD}$ protein band, which is supposed to be C-ADM140/MDA5 antibody; however, further determination was not available in our case. Arterial blood gas test at $\mathrm{O}_{2}$ inhalation of $3 \mathrm{~L} / \mathrm{min}$ with nasal cannula revealed decreased levels of $\mathrm{PaO}_{2}$ (68.7 Torr) and $\mathrm{PaCO}_{2}$ (31.7 Torr). Plasma b-type natriuretic peptide (BNP) was $<4.0 \mathrm{pg} / \mathrm{mL}[<18.4 \mathrm{pg} / \mathrm{mL}]$, and ultrasound cardiography revealed that his cardiac function was normal. Skin biopsy was performed, and the findings were compatible with dermatomyositis. Electromyogram or magnetic resonance imaging of thigh muscles was omitted because respiratory failure was progressing and the treatment was made the highest priority. There were no signs of malignancy so far as we could check.

He was diagnosed as clinically amyopathic dermatomyositis (C-ADM) with rapidly progressing interstitial pneumonia and treated with pulsed corticosteroids (methylprednisolone $1 \mathrm{~g} /$ day, days $0-3,16-18$ ) followed by high dose corticosteroids, intravenous cyclophosphamide (IVCY; $500 \mathrm{mg} / \mathrm{m}^{2}$, day 3,$22 ; 750 \mathrm{mg} / \mathrm{m}^{2}$, day 41 ), intravenous immunoglobulin (20 g/day, days 6-10, 48-52), and cyclosporine-A (CSA; $200 \mathrm{mg} /$ day, days 0-5; 250-300 mg/day, days 6-). Anticoagulation treatment with heparin followed by warfarin was also used (days 0-). In response to these initial treatments, consolidations and grand-glass opacities decreased in chest CT scans (Figure 2, day 7). Oxygen demand decreased from $4 \mathrm{~L} / \mathrm{min}$ to $1-2 \mathrm{~L} / \mathrm{min}$ to maintain $\mathrm{SpO}_{2}$ level at $90-95 \%$ in resting state. However, serum ferritin kept increasing and began to decrease two weeks afterwards.

On day 29, chest CT scans revealed progressing interstitial shadows again (Figure 2). CK was not elevated (130 U/L). Plasma BNP was slightly elevated $(26.9 \mathrm{pg} / \mathrm{mL})$, but there were no signs of heart failure. Although his oxygen demand or respiratory symptoms had not changed significantly, we
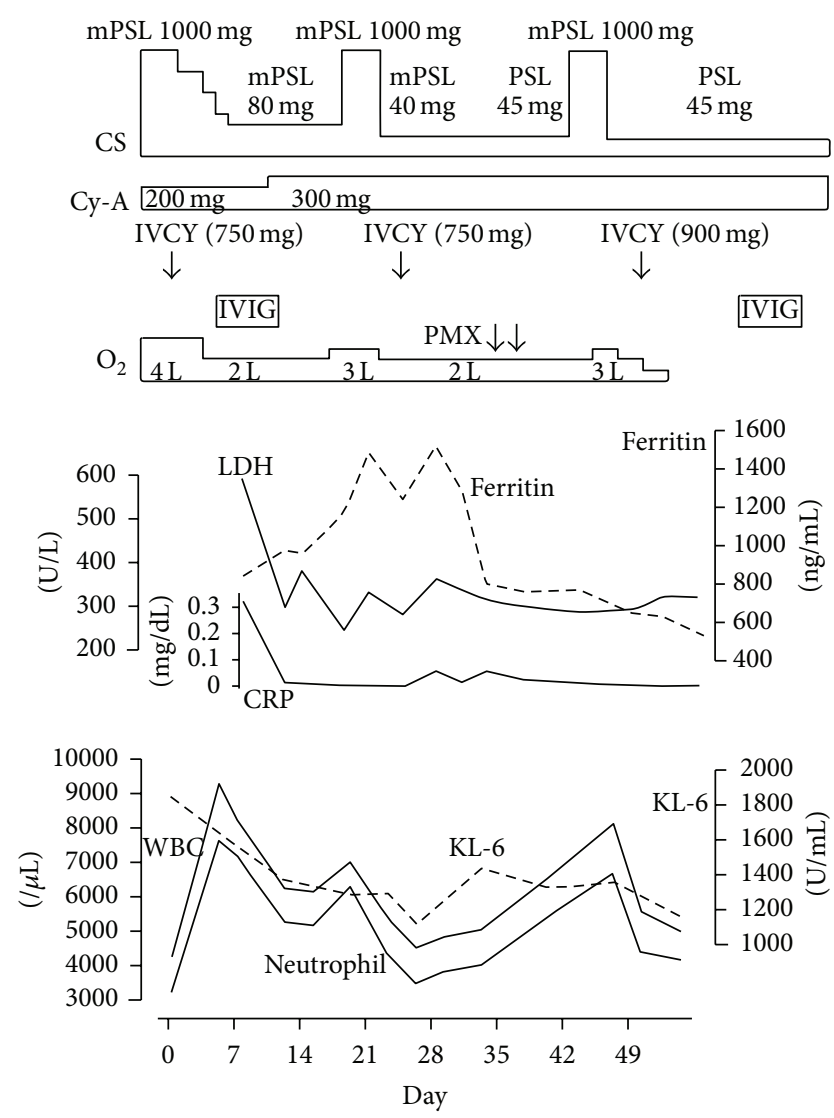

FIgURE 1: Time course of medical treatments and changes in the lab data before and after treatment. CS: corticosteroids; mPSL: intravenous methylprednisolone including pulse therapy; PSL: oral prednisolone; Cy-A: cyclosporine A; IVCY: intravenous cyclophosphamide therapy; IVIG: intravenous immunoglobulin $(20 \mathrm{~g} / \mathrm{d}$ for 5 days); PMX: polymyxin B-immobilized fiber column hemoperfusion; $\mathrm{O}_{2}$ : oxygen supplied with nasal cannula; $\mathrm{LDH}$ : serum lactate dehydrogenase; ferritin: serum ferritin level; CRP: serum C-reactive protein; KL-6: serum sialylated carbohydrate antigen KL-6 level; WBC: white blood cell count; neutrophil: neutrophil count.

thought that his interstitial lung disease was relapsing. Since a relapse after initial treatment of rapidly progressive ILD is often fatal, we decided to conduct intensive care in this early phase of the relapse, thus applied hemoperfusion treatment in this moment. On days 30 and 31, he underwent single course of PMX treatment, which was carried out at a flow rate of 80-100 $\mathrm{mL} / \mathrm{min}$ for $12 \mathrm{~h}$, twice at an interval of $24 \mathrm{~h}$. Nafamostat mesilate (Torii Co., Ltd, Tokyo, Japan) was used for anticoagulation. Following the hemoperfusion, the findings detected by chest X-ray and CT scans improved remarkably, and the serum ferritin kept decreasing. Oxygen demand improved gradually, reflected in the increase in arterial partial pressure of oxygen/fraction of inspired oxygen $\left(\mathrm{PaO}_{2} / \mathrm{FiO}_{2}\right)$ ratio (from 215 to 313), whereas serum concentration of LDH and KL-6 still remained at high level (Figure 1). The dosage of oral corticosteroid was tapered gradually, and he was discharged with a prescription of $30 \mathrm{mg}$ of prednisolone and $250 \mathrm{mg}$ of cyclosporine. He was treated with intravenous 
Day 0

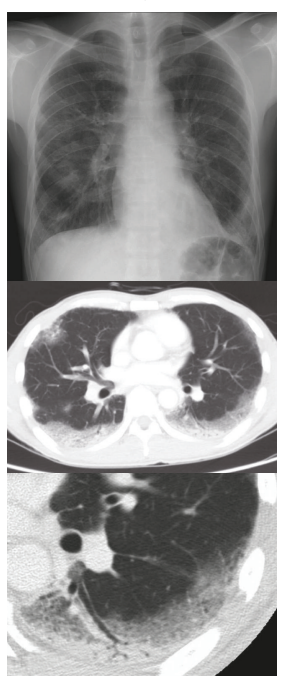

Day 29

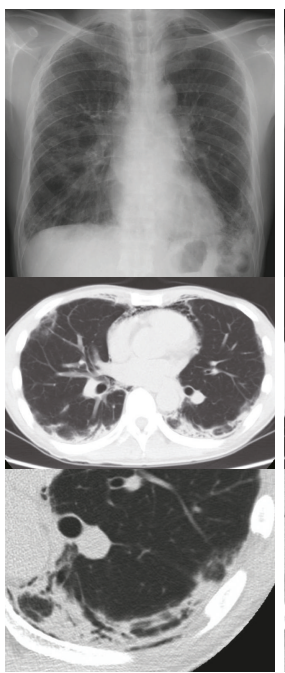

Day 7

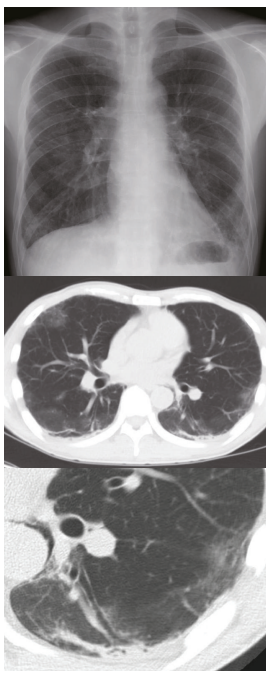

Day 33

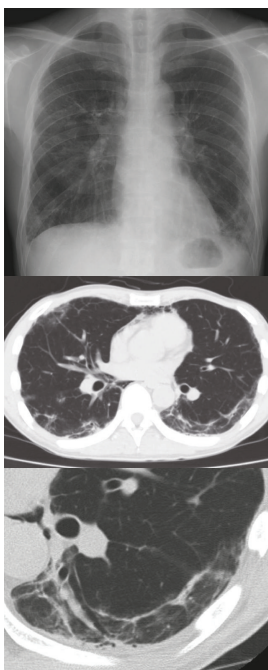

Day 15

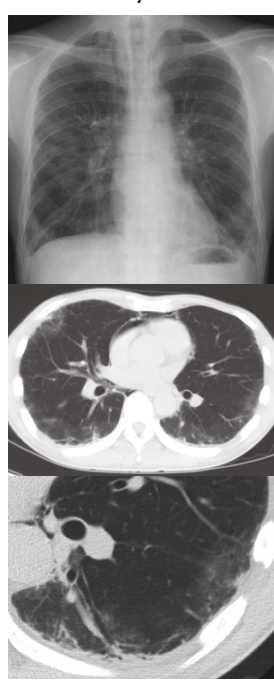

Day 50

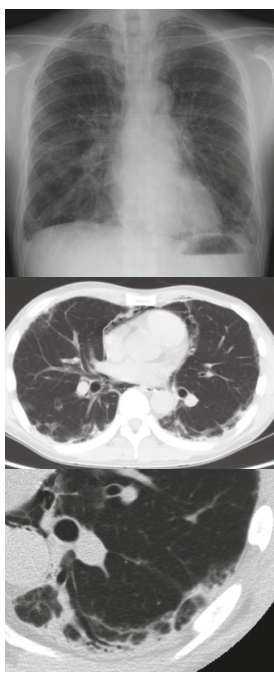

Figure 2: Chest X-rays and computed tomography (CT) scans of the patient with clinically amyopathic dermatomyositis (C$\mathrm{ADM}$ ) before and after polymyxin b-immobilized fiber column hemoperfusion (PMX) treatment. Day 0, on admission. Day 7, improvement after initial treatment. Day 15, before relapse. Day 29, on relapse and before PMX treatment. Day 33, improvement after PMX treatment (on days 30 and 31). Day 50, twenty days after PMX treatment.

cyclophosphamide repeatedly. There has been no recurrence in him for 6 months after the discharge.

\section{Discussion}

We report a case of rapidly progressive ILD with C-ADM, whose relapse in spite of intensive therapy was successfully prevented by PMX hemoperfusion. We think that PMX is a potent therapeutic option in combination with the conventional treatment, especially in the early phase of the relapse of the ILD with C-ADM.
Acute/subacute interstitial lung disease in patients with polymyositis/dermatomyositis (PM/DM) is frequently fatal within months despite high dose prednisolone (PSL) therapy [7]. Acute/subacute ILD is more common in patients with $\mathrm{C}-\mathrm{ADM}$, which results in higher mortality rate in these patients [8]. It is often resistant to conventional therapy and has poor prognosis especially in East Asia $[3,4]$. It was reported that HRCT patterns differed between anti-CADM-140 positive and negative patients in DM; lower lobes consolidation or ground-glass attenuation (GGA) pattern (50.0\%) and random GGA pattern (33.3\%) were the predominant patterns, and intralobular reticular opacities were absent in anti-C-ADM-140 positive cases, while lower lobes reticulation pattern (69.2\%) was frequently seen in anti-CADM-140 negative cases [9]. NSIP and DAD were seen in histological examinations of acute/subacute ILD with C-ADM [10]. Furthermore, C-ADM-associated interstitial pneumonia takes an aggressive course even when the radiological and histological features are consistent with NSIP [11]. In the present case, the radiological features were mostly consistent with lower lobes consolidation or GGA pattern, and there was traction bronchiectasis on admission, which is a predictor of rapid progression and poor prognosis of ILD [12]; therefore he received intensive initial treatment with pulsed corticosteroids and IVCY, intravenous immunoglobulin, oral CSA, and warfarin.

It was reported that combination therapy with pulsed corticosteroids, CSA, and IVCY was promising for acute/subacute ILD with DM [7]. It was reported that early intervention with CSA in combination with corticosteroid was effective, with tightly monitoring $2 \mathrm{~h}$ postdose blood concentration $(\mathrm{C} 2)$ of CSA $[4,13]$. In this case, we also used CSA as an initial treatment and monitored trough as well as C2 level of CSA. There are reports that intravenous immunoglobulin is effective for ILD with DM/PM [14, 15], and we also applied it twice with an interval of a month.

Although pulmonary coagulopathy is suspected to be involved in the pathogenesis of ILD, effectiveness of anticoagulation therapy for ILD is controversial [16-18]. As a maintenance therapy for idiopathic pulmonary fibrosis (IPF), warfarin had negative effect [16]. On the other hand, anticoagulation is known to be beneficial in acute exacerbation of various ILDs including IPF and C-ADM $[17,18]$. In our case, the patient presented with elevated plasma D-Dimer on admission, which might have indicated thrombus formation in pulmonary microcirculation, and we decided to begin anticoagulant therapy as an acute phase treatment.

In the present case, the intensive initial treatment with pulsed corticosteroids and cyclophosphamide twice, respectively, intravenous immunoglobulin once, and oral cyclosporine and warfarin succeeded in the induction of the remission to the rapidly progressing ILD with C-ADM. However, relapse was suspected soon after the initial treatment as progressing interstitial shadows without any signs of heart dysfunction. Although CT findings at the time of suspected relapse were not so prominent, we judged thatit was a critical time point to decide good or poor prognosis; thus we conducted polymyxin b-immobilized fiber column (PMX) treatment. 
PMX treatment was initially developed as a treatment for sepsis by removing circulating endotoxin. It has also revealed favorable effects on $\left(\mathrm{PaO}_{2} / \mathrm{FiO}_{2}\right)$ ratio in acute respiratory failure [6]. The number of reports is limited, but it was also effective for rapidly progressing ILD [19-21]. It has been reported that PMX treatment was also effective for ILD with C-ADM in combination with conventional treatment [2224]. Although there remains the possibility that the other immunosuppressants finally become effective, it seems that PMX played the major role in this case, since the progressing pulmonary infiltrates quickly cleared just after the PMX treatment.

However, the mechanism by which PMX improves pulmonary oxygenation in acute exacerbation of ILD (AEILD) has not been elucidated [25]. The removal of plasma endotoxin does not have a major role, because serum levels of endotoxin were within the normal range in previously reported cases of AE-ILD [19, 22, 25-27]. PMX treatment was known to reduce metalloproteinase- 9 and tissue inhibitor of metalloproteinase-1 [28], neutrophil reactive oxygen species [29], neutrophil elastase, and interleukin-8 [30]. These mediators cause lung injury and increase vascular permeability as well as the intrapulmonary shunt ratio. These mediators are known to be released from neutrophils and monocytes. It has been shown that the polymyxin b-immobilized fiber column itself traps activated blood neutrophils and monocytes, which may be involved in the reduction of releasing mediators [3133]. In our case, blood neutrophil count and monocyte count were not reduced after PMX treatment. The mechanism by which PMX treatment improved the ILD should be studied further. A long perfusion duration ( $12 \mathrm{~h}$ ) of PMX had better prognosis than short course $(<6 \mathrm{~h})$ for AE-ILD $[19,25]$, and we also applied $12 \mathrm{~h}$ for a hemoperfusion duration.

It has been reported that serum ferritin correlates with the activity of the ILD with C-ADM [34]. In our case, serum ferritin kept increasing for about a week after initiating intensive immunosuppressive therapies and began to decrease afterwards. There seemed to be a time lag of approximately two weeks between the decline of disease activity and decrease of the serum ferritin level.

KL-6 is a mucin-like glycoprotein expressed on type II pneumocytes. Serum KL-6 level has diagnostic and prognostic significance in ILD or acute respiratory distress syndrome $[35,36]$. It has better diagnostic accuracy of ILD in comparison to SP-A, SP-D, and MCP-1 [37, 38]. Serum KL6 is known to be elevated even 7-10 days after successful treatment $[39,40]$, which is compatible with our case.

Anticlinically amyopathic dermatomyositis- (C-ADM-) 140/MDA5 autoantibodies have been known to be a specific marker for C-ADM and strongly associated with rapidly progressive ILD with C-ADM [41]. Furthermore, their quantification may be useful for monitoring ILD activity [42].

In summary, we report a case of rapidly progressive interstitial pneumonia associated with C-ADM, whose relapse after intensive initial treatment was successfully prevented with single course of PMX hemoperfusion. PMX may be a potent therapeutic option in combination with conventional therapies for ILD associated with C-ADM, especially in the early phase of the relapse.

\section{References}

[1] P. Gerami, J. M. Schope, L. McDonald, H. W. Walling, and R. D. Sontheimer, "A systematic review of adult-onset clinically amyopathic dermatomyositis (dermatomyositis siné myositis): a missing link within the spectrum of the idiopathic inflammatory myopathies," Journal of the American Academy of Dermatology, vol. 54, no. 4, pp. 597-613, 2006.

[2] M. Kalluri and C. V. Oddis, "Pulmonary manifestations of the idiopathic inflammatory myopathies," Clinics in Chest Medicine, vol. 31, no. 3, pp. 501-512, 2010.

[3] S. Sato and M. Kuwana, "Clinically amyopathic dermatomyositis," Current Opinion in Rheumatology, vol. 22, no. 6, pp. 639643, 2010.

[4] T. Kotani, T. Takeuchi, S. Makino et al., "Combination with corticosteroids and cyclosporin-A improves pulmonary function test results and chest HRCT findings in dermatomyositis patients with acute/subacute interstitial pneumonia," Clinical Rheumatology, vol. 30, no. 8, pp. 1021-1028, 2011.

[5] H. Shoji, "Extracorporeal endotoxin removal for the treatment of sepsis: endotoxin adsorption cartridge (Toraymyxin)," Therapeutic Apheresis and Dialysis, vol. 7, no. 1, pp. 108-114, 2003.

[6] D. N. Cruz, M. A. Perazella, R. Bellomo et al., "Effectiveness of polymyxin B-immobilized fiber column in sepsis: a systematic review," Critical Care, vol. 11, article R47, 2007.

[7] H. Kameda, H. Nagasawa, H. Ogawa et al., "Combination therapy with corticosteroids, cyclosporin A, and intravenous pulse cyclophosphamide for acute/subacute interstitial pneumonia in patients with dermatomyositis," Journal of Rheumatology, vol. 32, no. 9, pp. 1719-1726, 2005.

[8] H. Mukae, H. Ishimoto, N. Sakamoto et al., "Clinical differences between interstitial lung disease associated with clinically amyopathic dermatomyositis and classic dermatomyositis," Chest, vol. 136, no. 5, pp. 1341-1347, 2009.

[9] K. Tanizawa, T. Handa, R. Nakashima et al., "HRCT features of interstitial lung disease in dermatomyositis with anti-CADM140 antibody," Respiratory Medicine, vol. 105, no. 9, pp. 13801387, 2011.

[10] T. Suda, T. Fujisawa, N. Enomoto et al., "Interstitial lung diseases associated with amyopathic dermatomyositis," European Respiratory Journal, vol. 28, no. 5, pp. 1005-1012, 2006.

[11] E. Miyazaki, M. Ando, T. Muramatsu et al., "Early assessment of rapidly progressive interstitial pneumonia associated with amyopathic dermatomyositis," Clinical Rheumatology, vol. 26, no. 3, pp. 436-439, 2007.

[12] G. Ideura, M. Hanaoka, T. Koizumi et al., "Interstitial lung disease associated with amyopathic dermatomyositis: review of 18 cases," Respiratory Medicine, vol. 101, no. 7, pp. 1406-1411, 2007.

[13] T. Kotani, S. Makino, T. Takeuchi et al., "Early intervention with corticosteroids and cyclosporin A and 2-hour postdose blood concentration monitoring improves the prognosis of acute/subacute interstitial pneumonia in dermatomyositis," Journal of Rheumatology, vol. 35, no. 2, pp. 254-259, 2008.

[14] Y. Suzuki, H. Hayakawa, S. Miwa et al., "Intravenous immunoglobulin therapy for refractory interstitial lung disease associated with polymyositis/dermatomyositis," Lung, vol. 187, no. 3, pp. 201-206, 2009.

[15] C. J. Bakewell and G. Raghu, "Polymyositis associated with severe interstitial lung disease: remission after three doses of IV immunoglobulin," Chest, vol. 139, no. 2, pp. 441-443, 2011. 
[16] I. Noth, K. J. Anstrom, S. B. Calvert et al., "A placebo-controlled randomized trial of warfarin in idiopathic pulmonary fibrosis," American Journal of Respiratory and Critical Care Medicine, vol. 186, no. 1, pp. 88-95, 2012.

[17] H. Kubo, K. Nakayama, M. Yanai et al., "Anticoagulant therapy for idiopathic pulmonary fibrosis," Chest, vol. 128, no. 3, pp. 1475-1482, 2005.

[18] K. Watanabe, S. Tajima, J. Tanaka et al., "Effects of anticoagulant therapy for rapidly progressive interstitial pneumonias," Nihon Kokyuki Gakkai Zasshi, vol. 49, no. 6, pp. 407-412, 2011.

[19] N. Enomoto, T. Suda, T. Uto et al., "Possible therapeutic effect of direct haemoperfusion with a polymyxin B immobilized fibre column (PMX-DHP) on pulmonary oxygenation in acute exacerbations of interstitial pneumonia," Respirology, vol. 13, no. 3, pp. 452-460, 2008.

[20] Y. Sakamoto, K. Mashiko, T. Obata et al., "Relationship between treatment resistance to hemoperfusion using a polymyxin bimmobilized fiber column and oxidative stress," ASAIO Journal, vol. 54, no. 4, pp. 412-415, 2008.

[21] S. Hara, H. Ishimoto, N. Sakamoto et al., "Direct hemoperfusion using immobilized polymyxin B in patients with rapidly progressive interstitial pneumonias: a retrospective study," Respiration, vol. 81, no. 2, pp. 107-117, 2011.

[22] T. Kakugawa, H. Mukae, M. Saito et al., "Rapidly progressive interstitial pneumonia associated with clinically amyopathic dermatomyositis successfully treated with polymyxin Bimmobilized fiber column hemoperfusion," Internal Medicine, vol. 47, no. 8, pp. 785-790, 2008.

[23] H. Ichiyasu, Y. Horio, S. Tsumura et al., "Favorable outcome with hemoperfusion of polymyxin B-immobilized fiber column for rapidly progressive interstitial pneumonia associated with clinically amyopathic dermatomyositis: report of three cases," Modern Rheumatology, 2012.

[24] S. Abe, A. Azuma, H. Mukae et al., "Polymyxin B-immobilized fiber column (PMX) treatment for idiopathic pulmonary fibrosis with acute exacerbation: a multicenter retrospective analysis," Internal Medicine, vol. 51, no. 12, pp. 1487-1491, 2012.

[25] M. Kono, T. Suda, N. Enomoto et al., "Evaluation of different perfusion durations in direct hemoperfusion with polymyxin B-immobilized fiber column therapy for acute exacerbation of interstitial pneumonias," Blood Purification, vol. 32, no. 2, pp. 75-81, 2011.

[26] Y. Seo, S. Abe, M. Kurahara et al., "Beneficial effect of polymyxin B-immobilized fiber column (PMX) hemoperfusion treatment on acute exacerbation of idiopathic pulmonary fibrosis," Internal Medicine, vol. 45, no. 18, pp. 1033-1038, 2006.

[27] S. Noma, W. Matsuyama, H. Matsuyama et al., "Two cases of acute exacerbation of interstitial pneumonia treated with polymyxin B-immobilized fiber column hemoperfusion treatment," Internal Medicine, vol. 46, no. 17, pp. 1447-1454, 2007.

[28] T. Nakamura, Y. Kawagoe, T. Matsuda et al., "Effect of polymyxin B-immobilized fiber on blood metalloproteinase9 and tissue inhibitor of metalloproteinase-1 levels in acute respiratory distress syndrome patients," Blood Purification, vol. 22, no. 3, pp. 256-260, 2004.

[29] T. Naka, M. Shinozaki, T. Akizawa, Y. Shima, H. Takaesu, and H. Nasu, "The effect of continuous veno-venous hemofiltration or direct hemoperfusion with polymyxin B-immobilized fiber on neutrophil respiratory oxidative burst in patients with sepsis and septic shock," Therapeutic Apheresis and Dialysis, vol. 10, no. 1, pp. 7-11, 2006.
[30] H. Kushi, T. Miki, K. Okamaoto, J. Nakahara, T. Saito, and K. Tanjoh, "Early hemoperfusion with an immobilized polymyxin B fiber column eliminates humoral mediators and improves pulmonary oxygenation," Critical Care, vol. 9, no. 6, pp. R653R661, 2005.

[31] H. Tsujimoto, S. Ono, S. Hiraki et al., "Hemoperfusion with polymyxin B-immobilized fibers reduced the number of CD16+CD14+ monocytes in patients with septic shock," Journal of Endotoxin Research, vol. 10, no. 4, pp. 229-237, 2004.

[32] S. Ono, H. Tsujimoto, A. Matsumoto, S. Ikuta, M. Kinoshita, and H. Mochizuki, "Modulation of human leukocyte antigen-DR on monocytes and CD16 on granulocytes in patients with septic shock using hemoperfusion with polymyxin B-immobilized fiber," American Journal of Surgery, vol. 188, no. 2, pp. 150-156, 2004.

[33] S. Abe, Y. Seo, H. Hayashi et al., "Neutrophil adsorption by polymyxin b-immobilized fiber column for acute exacerbation in patients with interstitial pneumonia: a pilot study," Blood Purification, vol. 29, no. 4, pp. 321-326, 2010.

[34] T. Gono, Y. Kawaguchi, E. Ozeki et al., "Serum ferritin correlates with activity of anti-MDA5 antibody-associated acute interstitial lung disease as a complication of dermatomyositis," Modern Rheumatology, vol. 21, no. 2, pp. 223-227, 2011.

[35] A. Ishizaka, T. Matsuda, K. H. Albertine et al., "Elevation of KL6, a lung epithelial cell marker, in plasma and epithelial lining fluid in acute respiratory distress syndrome," American Journal of Physiology, vol. 286, no. 6, pp. L1088-L1094, 2004.

[36] H. Sato, M. E. J. Callister, S. Mumby et al., "KL-6 levels are elevated in plasma from patients with acute respiratory distress syndrome," European Respiratory Journal, vol. 23, no. 1, pp. 142$145,2004$.

[37] H. Ohnishi, A. Yokoyama, K. Kondo et al., "Comparative study of KL-6, surfactant protein-A, surfactantprotein-D, and monocyte chemoattractant protein-1 as serum markersfor interstitial lung diseases," American Journal of Respiratory and Critical Care Medicine, vol. 165, no. 3, pp. 378-381, 2002.

[38] N. Nathani, G. D. Perkins, W. Tunnicliffe, N. Murphy, M. Manji, and D. R. Thickett, "Kerbs von Lungren 6 antigen is a marker of alveolar inflammation but not of infection in patients with acute respiratory distress syndrome," Critical Care, vol. 12, no. 1, article R12, 2008.

[39] M. Otsuka, H. Takahashi, T. Fujisima et al., "New serum markers to monitor treatment of acute exacerbation of interstitial lung disease," Nihon Kokyuki Gakkai Zasshi, vol. 39, no. 4, pp. 298-302, 2001.

[40] A. Sadakata, M. Yamaguchi, Y. Misaki et al., "Acute exacerbation of interstitial pneumonia associated with active systemic lupus erythematosus in a patient with rheumatoid arthritis," Nihon Kokyuki Gakkai Zasshi, vol. 40, no. 9, pp. 756-761, 2002.

[41] S. Sato, M. Hirakata, M. Kuwana et al., "Autoantibodies to a 140kd polypeptide, CADM-140, in Japanese patients with clinically amyopathic dermatomyositis," Arthritis and Rheumatism, vol. 52, no. 5, pp. 1571-1576, 2005.

[42] S. Sato, M. Kuwana, T. Fujita, and Y. Suzuki, "Amyopathic dermatomyositis developing rapidly progressive interstitial lung disease with elevation of anti-CADM-140/MDA5 autoantibodies," Modern Rheumatology, vol. 22, no. 4, pp. 625-629, 2012. 


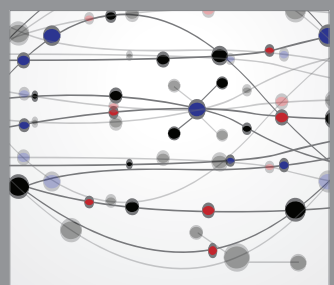

The Scientific World Journal
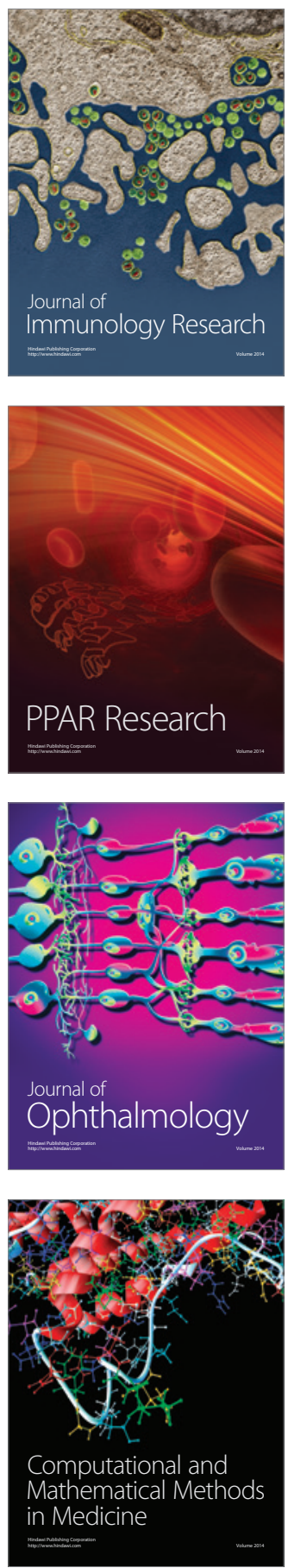

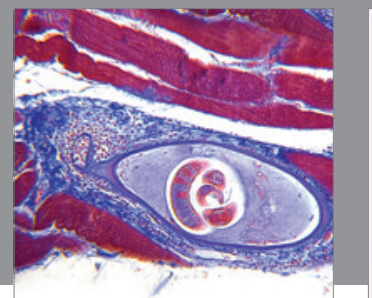

Gastroenterology

Research and Practice
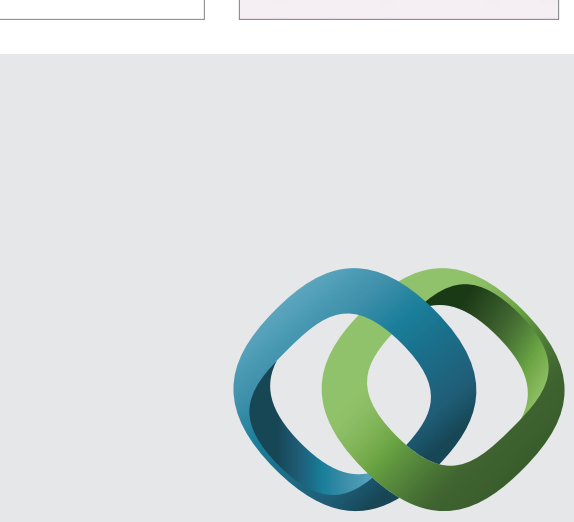

\section{Hindawi}

Submit your manuscripts at

http://www.hindawi.com
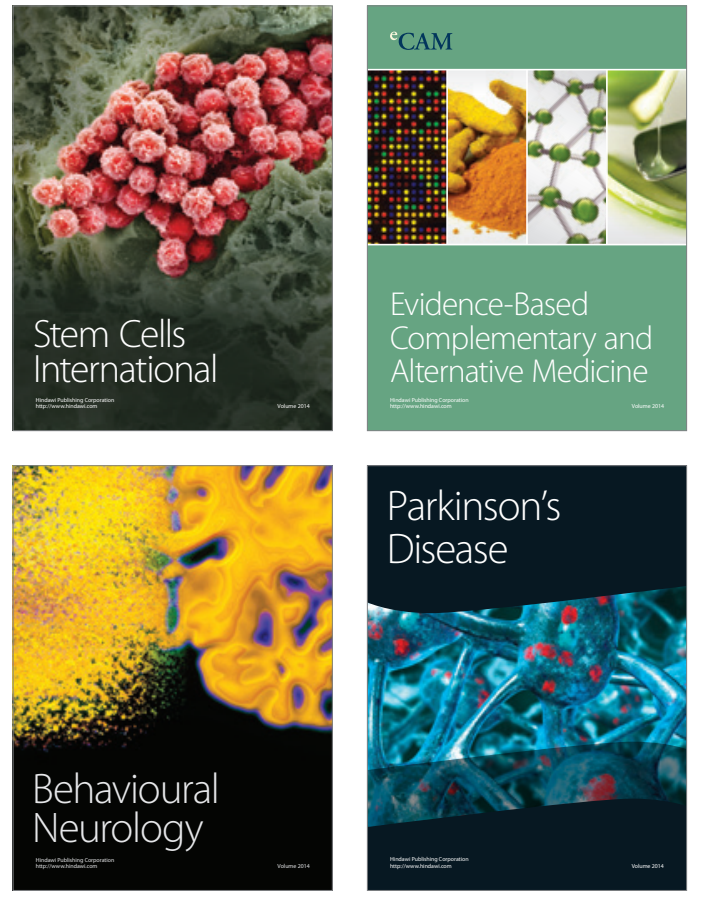
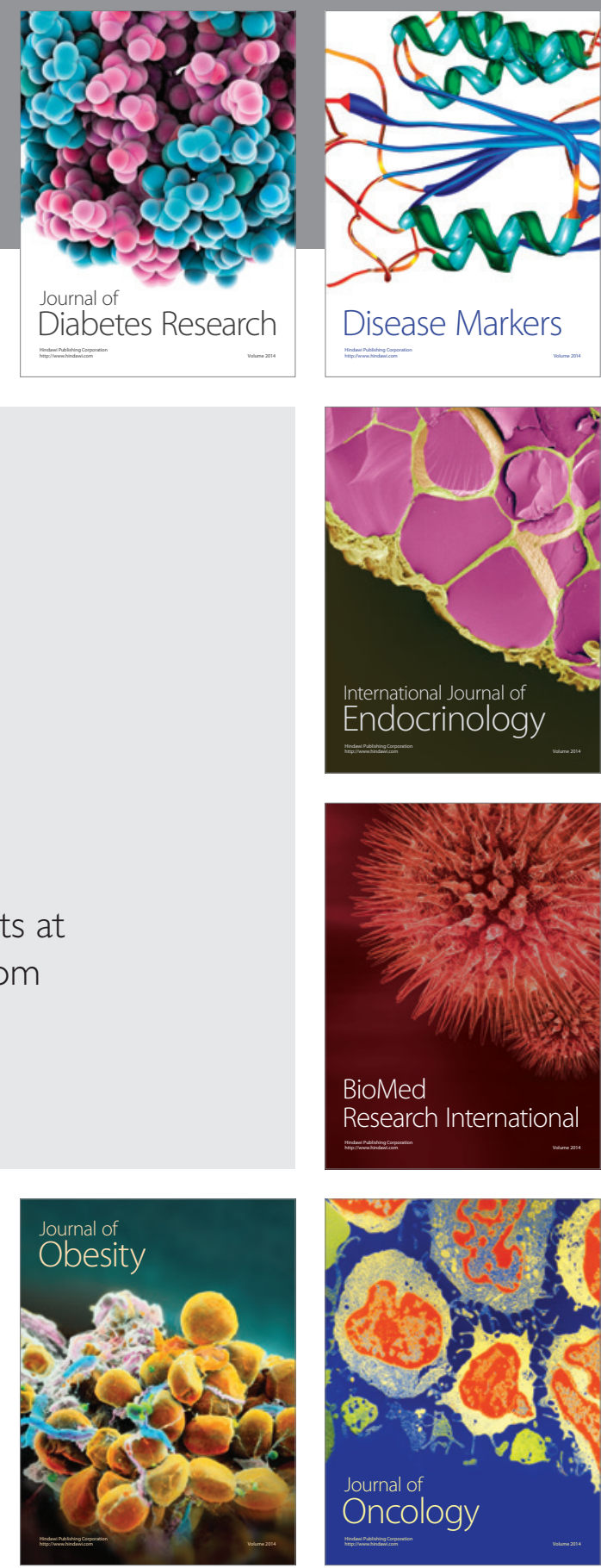

Disease Markers
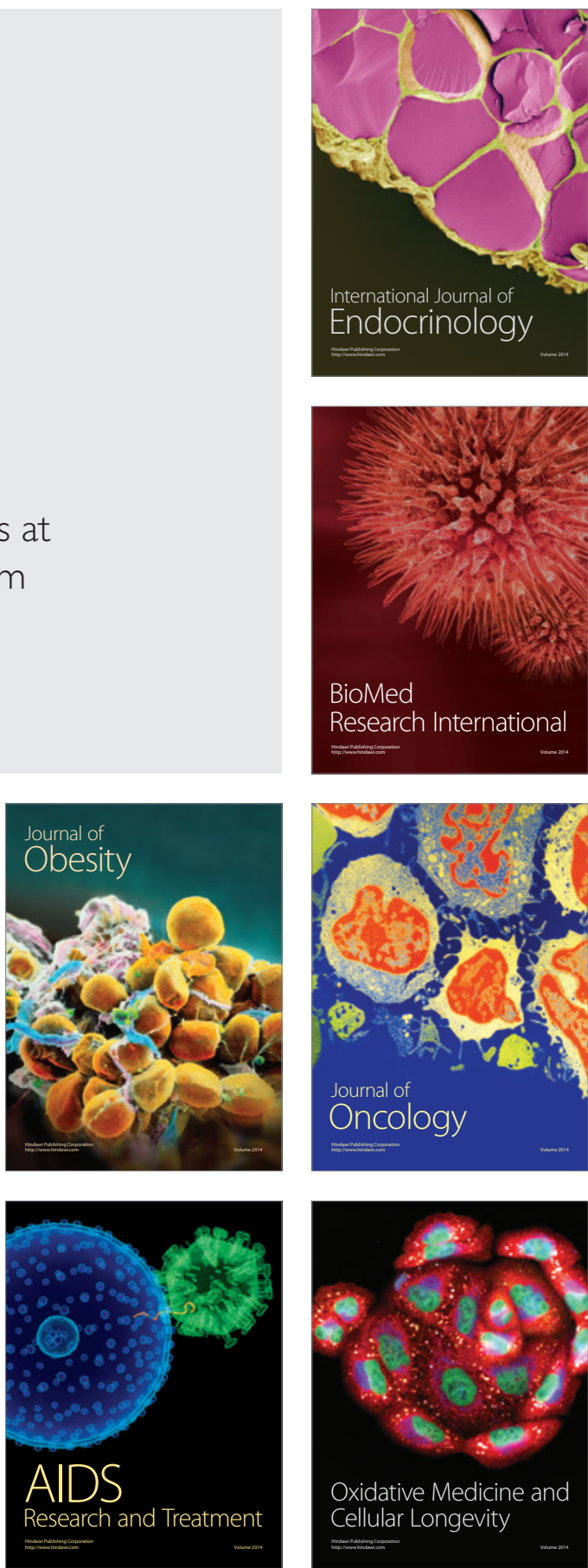